\title{
Ultrabroadband Nonlinear Optics in Dispersion Engineered Periodically Poled Lithium Niobate Waveguides
}

\author{
Marc Jankowski*, $^{* 1}$, Carsten Langrock ${ }^{1}$, Boris Desiatov ${ }^{2}$, Alireza Marandi ${ }^{3}$, Cheng \\ Wang $^{2}$, Mian Zhang ${ }^{2}$, Chris R. Phillips ${ }^{4}$, Marko Loncar ${ }^{2}$, Martin M. Fejer ${ }^{1}$ \\ ${ }^{1}$ Edward L. Ginzton Laboratory, Stanford University, Stanford, CA, 94305, USA \\ ${ }^{2}$ John A. Paulson School of Engineering and Applied Sciences, Harvard University, Cambridge, Massachusetts \\ 02138, USA \\ ${ }^{3}$ California Institute of Technology, Pasadena, CA 91125, USA \\ ${ }^{4}$ Department of Physics, Institute of Quantum Electronics, ETH Zurich, Zurich 8093, Switzerland \\ *marcjank@stanford.edu
}

\begin{abstract}
We experimentally demonstrate the first generation of dispersion-engineered periodically poled lithium niobate (PPLN) waveguides. These waveguides achieve ultrabroadband second-harmonic generation (SHG) and multi-octave supercontinuum generation (SCG) with record-low pulse energies. @ 2019 The Author(s)
\end{abstract}

OCIS codes: (190.4390) Nonlinear Optics, Integrated Optics; (190.2620) Nonlinear Optics, Harmonic Generation and Mixing; (320.6629) Ultrafast Optics, Supercontinuum Generation

\section{Introduction}

The current state of the art of nonlinear guided-wave devices comprise two approaches: $\chi^{(3)}$ interactions in tightlyconfining dispersion-engineered waveguides [1], and quasi-phasematched $\chi^{(2)}$ interactions in periodically poled waveguides $[2,3]$. While the operating regimes of $\chi^{(3)}$ devices can be controlled by a suitable choice of waveguide group velocity dispersion, such devices are ultimately limited to soliton formation, spectral broadening, and, in phase-velocity engineered waveguides, non-degenerate four-wave mixing. A broader range of nonlinear processes can be accessed in periodically poled $\chi^{(2)}$ waveguides, where the desired process can be phasematched with a suitable choice of poling period. The bandwidths (and hence useful lengths for pulsed interactions) of these processes have ultimately been limited by the material dispersion that dominates in conventional loosely confining waveguides. Recent work has combined high-fidelity periodic poling with low-loss tightly confining waveguides to achieve record-high normalized conversion efficiencies $\left(2000 \% / \mathrm{W}-\mathrm{cm}^{2}\right)$ [4], but until now dispersionengineered quasi-phasematched $\chi^{(2)}$ interactions have not been demonstrated. In this work we use direct-etched nanophotonic PPLN ridge waveguides to provide the first experimental demonstration of ultra-broadband quasiphasematched $\chi^{(2)}$ interactions in a dispersion-engineered waveguide. These devices, which have been designed for broadband SHG of wavelengths around 2- $\mu \mathrm{m}$, exhibit SHG transfer functions with bandwidths $>250 \mathrm{~nm}$. Furthermore, these waveguides achieve saturated SHG with pulse energies as low as $1 \mathrm{pJ}$ when pumped with $\sim 50$-fs-long pulses around $2 \mu \mathrm{m}$. When driven with pulse energies in excess of a $\mathrm{pJ}$, these waveguides exhibit a cascade of mixing processes and spectral broadening, resulting in a supercontinuum spanning $\sim 2.5$ octaves at the $-25 \mathrm{~dB}$ level with $53 \mathrm{pJ}$ of input power.
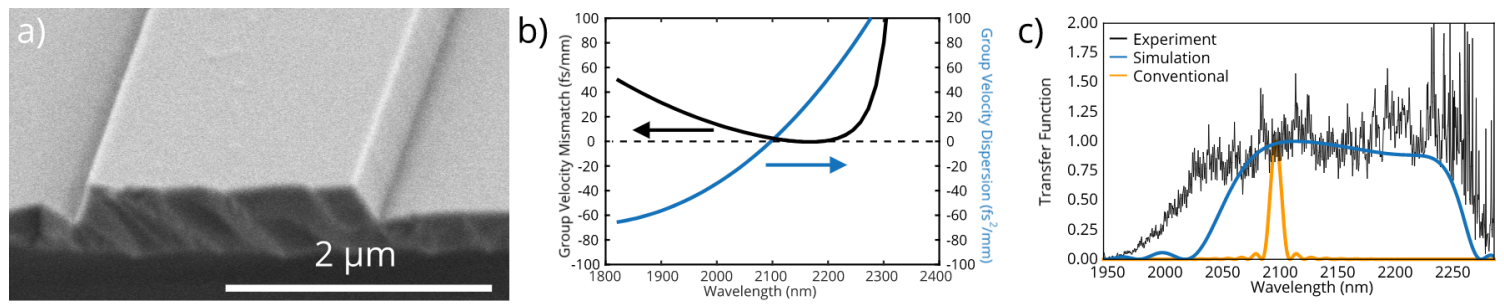

Fig. 1. a) SEM image of a dispersion-engineered PPLN waveguide. b) Simulated $\Delta \beta^{\prime}$ and $\beta_{\omega}^{\prime \prime}$ showing group-velocity matching and a zero dispersion wavelength around 2- $\mu \mathrm{m} . \mathrm{c})$ Measured and theoretical SHG transfer function showing a bandwidth $\sim 250 \mathrm{~nm}$. These devices exceed the bandwidth of conventional weakly guiding PPLN waveguides of the same length by an order of magnitude. 


\section{Results}

The bandwidth of SHG interactions in weakly guiding waveguides is dominated by the group-velocity mismatch $\left(\Delta \beta^{\prime}=\beta_{2 \omega}^{\prime}-\beta_{\omega}^{\prime}\right)$ between the fundamental and second harmonic, with corresponding scaling law $\Delta \lambda \propto 1 /\left|\Delta \beta^{\prime}\right| L$. Ultra-broadband interactions become possible when the geometric dispersion of tightly confining waveguide achieves $\Delta \beta^{\prime}=0$. In this case the corresponding SHG bandwidth becomes dominated by higher order dispersion, and scales more slowly with device length. Figure 1(a) shows a scanning electron microscope (SEM) image of a PPLN waveguide fabricated for broadband SHG of wavelengths around 2-um. The simulated $\Delta \beta^{\prime}$ as well as the group-velocity dispersion of the fundamental $\left(\beta_{\omega}^{\prime \prime}\right)$ are plotted in Fig. 1(b), with the waveguide geometry chosen such that both $\Delta \beta^{\prime} \approx 0$ and $\beta_{\omega}^{\prime \prime} \approx 0$. We characterize the SHG transfer function using $\sim 50$-fs-long pulses from a synchronously pumped degenerate optical parametric oscillator. Figure 1(c) shows the calculated and measured SHG transfer function for a 6-mm-long device, indicating a bandwidth $>250 \mathrm{~nm}$, which outperforms bulk $2-\mu \mathrm{m}$ SHG devices of the same length in PPLN by an order of magnitude.
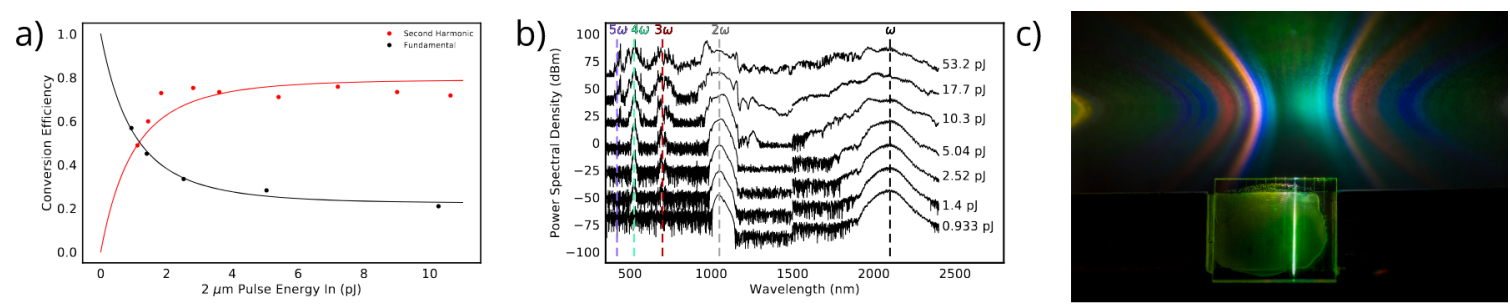

Fig. 2. a) SHG conversion efficiency and pump depletion as a function of input pulse energy. b) Power spectral density output from the chip as a function of input pulse energy, showing the generation of multiple harmonics, and SCG for pulse energies $>10$ pJ. c) Photograph of SCG produced with $\sim 53-\mathrm{pJ}$ input pulses. The observed diffraction pattern is due to quasi-periodic facet corrugation seen in Fig. 1(a), which act as a diffraction grating.

The conversion efficiency of the waveguide as a function of input pulse energy is shown in Fig. 2(a). Saturation occurs for pulse energies as low as 1-pJ, which is an order of magnitude larger than anticipated from theoretical modeling. Previous devices fabricated similarly to these showed continuous-wave slope efficiencies within a factor of two of simulations [4], and the origin of this discrepancy in poling errors or in excess loss is under study. The conversion efficiency is clamped at $75 \%$ due to parasitic mixing processes, which lead to the generation of the $3 \mathrm{rd}, 4 \mathrm{th}$, and 5 th harmonic for pulse energies greater than $2.5 \mathrm{pJ}$. When the waveguide is driven far into saturation $(>10 \mathrm{pJ})$ the harmonics undergo spectral broadening, and generate a supercontinuum spanning $\sim 2.5$ octaves at the $-25 \mathrm{~dB}$ level for an input pulse energy of $53 \mathrm{pJ}$. The measured supercontinuum is limited to wavelengths $>400$ $\mathrm{nm}$ by the transparency window of our collection optics, and $<2400 \mathrm{~nm}$ by our available spectrometers. Past demonstrations of multi-octave SCG in PPLN waveguides have required input pulse energies $>1 \mathrm{~nJ}$ [3].

\section{Conclusion}

We have experimentally demonstrated both SHG and SCG in a dispersion-engineered PPLN waveguide. These waveguides are shown to exceed the performance of current-generation SHG devices by at least an order of magnitude in phasematching bandwidth and pulse energy requirements. Similarly, they achieve multi-octave SCG with an order of magnitude less pulse energy than conventional PPLN devices. Future work will include a detailed characterization of the SHG slope efficiency, and the coherence of the generated supercontinuum. Resolution of the discrepancy between experiment and simulation is expected to yield an order of magnitude reduction in the required pulse energies. Such devices will find use as integrated comb sources, f- $2 \mathrm{f}$ interferometers, parametric amplifiers, and quantum frequency converters.

\section{References}

1. A. S. Mayer, et al, "Frequency comb offset detection using supercontinuum generation in silicon nitride waveguides," Opt. Express 23, 15440-15451 (2015).

2. A. S. Mayer, et al, "Offset-Free Gigahertz Midinfrared Frequency Comb Based on Optical Parametric Amplification in a Periodically Poled Lithium Niobate Waveguide," Phys. Rev. Applied 6, 054009 (2016)

3. C. R. Phillips, et al, "Supercontinuum generation in quasi-phase-matched LiNbO3 waveguide pumped by a Tm-doped fiber laser system," Opt. Lett. 19, 3912 (2011).

4. C. Wang, et al, "Ultrahigh-efficiency wavelength conversion in nanophotonic periodically poled lithium niobate waveguides," Optica 5, 1438-1441 (2018). 